\title{
EchoGéo
}

$11 \mid 2010$

Madagascar

\section{La Politique Antillaise du Président Sarkozy}

Des équivoques du passé aux convergences du présent

\section{Fred Constant}

\section{(2) OpenEdition}

Journals

Édition électronique

URL : https://journals.openedition.org/echogeo/11719

DOI : 10.4000/echogeo. 11719

ISSN : 1963-1197

Éditeur

Pôle de recherche pour l'organisation et la diffusion de l'information géographique (CNRS UMR 8586)

Référence électronique

Fred Constant, «La Politique Antillaise du Président Sarkozy », EchoGéo [En ligne], 11 | 2010, mis en ligne le 24 février 2010, consulté le 01 août 2021. URL : http://journals.openedition.org/echogeo/ 11719 ; DOI : https://doi.org/10.4000/echogeo.11719

Ce document a été généré automatiquement le 1 août 2021.

EchoGéo est mis à disposition selon les termes de la licence Creative Commons Attribution - Pas d'Utilisation Commerciale - Pas de Modification 4.0 International (CC BY-NC-ND) 


\section{La Politique Antillaise du Président Sarkozy}

Des équivoques du passé aux convergences du présent

\section{Fred Constant}

1 Jeudi 19 février 2009. Palais de l'Elysée. Le président de la République reçoit les présidents des collectivités locales et les parlementaires des départements d'Outre-mer. En toile de fond, une crise sociale, identitaire et politique sans précédent (Daniel, 2009). Pendant près d'un mois, l'impuissance des élus locaux fait face au silence assourdissant $\mathrm{du}$ gouvernement. Sur le terrain, le désordre et la violence gagnent tandis que les partenaires sociaux peinent à engager le dialogue. Les collectifs syndicaux du LKP et du 5 février s'impatientent face au blocage des négociations et au désarroi des responsables politiques. Dans les mêmes termes, ils dénoncent le mépris sinon l'indifférence de l'Etat. Les tentatives de médiation du Secrétaire d'Etat à l'Outre-mer ne changent rien sur le fond. Le climat social reste lourd. La mort tragique d'un syndicaliste, en Guadeloupe, emporte le risque d'une généralisation de la violence alors que l'afflux des forces de maintien de l'ordre y atteint - ainsi qu'en Martinique - des niveaux historiques. Dans les deux îles, le spectre du passé revient au premier plan des consciences : fin décembre 1959 à Fort-de-France, 25-27 mai 1967 à Pointe-à-Pitre. Deux révoltes populaires durement réprimées dans le sang...

Quand il entre dans le salon Murat, accompagné par le Premier ministre et le ministre de l'Intérieur, de l'Outre-mer et des collectivités locales, le chef de l'Etat a pleinement conscience de la situation très préoccupante des Antilles - où sa côte de popularité est plutôt faible - mais aussi du bénéfice politique qu'il pourrait tirer d'un retournement de la situation à son avantage. Dans son intervention liminaire ${ }^{1}$, le président de la République fait part aux élus de son analyse de la crise avant d'annoncer quelques mesures susceptibles de répondre aux préoccupations immédiates en matière de pouvoir d'achat et d'emploi. Mais soucieux de reprendre l'initiative politique, il saisit l'occasion pour jeter les bases d'une réflexion de fond sur l'avenir des territoires d'Outre-mer, en posant sans détour les trois questions qui hantent l'histoire des "quatre vieilles colonies françaises»: quel type de développement économique et 
social ? Quel modèle d'organisation politique ? Quelle relation avec la métropole ? Pour y répondre, il choisit la méthode inédite des Etats généraux de l'Outre-mer (EGOM) dont il s'engage à donner suite aux propositions à l'occasion du tout premier Conseil interministériel de l'Outre-mer (CIOM) prévu en octobre prochain.

Lors de sa tournée antillaise de juin 2009, le chef de l'Etat n'a toutefois pas manqué de lever un coin du voile sur le nouveau cap de l'action gouvernementale Outre-mer, préemptant malgré ses précautions oratoires certaines des futures décisions du CIOM. Ce nouveau cap repose sur quatre idées centrales: a) rejet du statu quo ante et des ajustements marginaux b) évolutions institutionnelles - ou statutaires - locales et adaptation des administrations centrales c) promotion d'un modèle de développement économique endogène et durable d) reconnaissance de la singularité irréductible des Outre-mers et de la diversité des aspirations politiques de ses composantes. En martelant ses nouvelles convictions, le président ne cherche pas seulement à gommer les malentendus du passé ${ }^{2}$ en esquissant des réponses aux défis du présent. A l'évidence, il entend autant faire bouger les lignes du débat public Outre-mer pour peser davantage sur le jeu politique insulaire. A plus d'un titre, la Martinique et la Guadeloupe offrent une expression exemplaire sinon illustrative des changements en cours.

\section{Evolution institutionnelle (art. 73) ou statutaire (art. 74) ?}

4 «Je crois que nous sommes arrivés à la fin d'un cycle historique en outre-mer. Nous n'apporterons pas de réponses appropriées si nous nous contentons d'accumuler des annonces financières et techniques $»^{3}$. En deux phrases, le chef de l'Etat reprend à son compte le diagnostic maintes fois réitéré par les élus locaux d'un système départemental « essoufflé » et valide les démarches engagées par ces derniers en faveur d'un nouveau schéma de partage des responsabilités avec l'Etat. Dans son discours de baptême de l'aéroport Aimé Césaire, le président va plus loin en évoquant les différentes options ouvertes par la constitution: de la collectivité unique soumise au principe d'identité législative mais dotée d'un pouvoir normatif renforcé (article 73) au statut de collectivité d'Outre-mer soumise au principe de spécialité législative et dotée d'une autonomie normative et administrative accrue par rapport à la métropole (article 74). Lors de son intervention dans l'île sœur, il réitère sa conviction de la nécessité d'adapter la gouvernance - locale et centrale - pour répondre plus efficacement aux défis d'un développement économique plus endogène. Dans les deux départements, il déroute ses alliés autant qu'il séduit ses opposants. En se gardant bien de trancher le débat, il n'hésite pas à marginaliser le conservatisme des premiers et à banaliser «l'autonomisme» des seconds.

5 Cette évolution ne s'est pourtant pas faite ex nihilo (Constant, 2001). Elle a été rendue possible par la levée progressive du tabou sur la question du statut et l'affirmation corollaire du principe d'évolution institutionnelle différenciée des Outre-mers. Formulé pour la première fois en 1988 par Louis Le Pensec, alors Ministre de l'Outre-mer, et réaffirmé par Lionel Jospin lors de la campagne des élections législatives en 1997, ce principe a été conforté par la « déclaration de Basse-Terre » du $1^{\text {er }}$ décembre 1999, le rapport parlementaire Lise-Tamaya (1999) et le vote de la loi du 13 décembre 2000. En 2003, la mise en œuvre de la procédure du congrès a donné lieu, pour la première fois, à 
la consultation des populations en Martinique et en Guadeloupe. L'échec de celle-ci a suspendu provisoirement la question institutionnelle qui devait cependant être relancée en 2008 avec la transformation de Saint-Barthélemy et Saint-Martin en collectivités d'Outre-mer régies par l'article 74. Pour l'heure, seuls les élus de Guyane et de Martinique ont engagé la procédure du congrès en vue d'adopter un projet de collectivité régie par l'article 74 . Le calendrier initial a cependant été fortement perturbé par la « crise sociale » de février 2009 qui a déstabilisé les exécutifs locaux et pris de court le Gouvernement de François Fillon. En Guadeloupe, les présidents des assemblées départementale et régionale ont décidé de prendre le temps de la réflexion. La question de l'évolution institutionnelle ou statutaire ne leur semble plus prioritaire. L'heure est plutôt à l'apaisement et à la réduction des tensions sociales.

6 Cette individuation des trajectoires politiques gomme la symétrie des évolutions du passé. Des deux côtés de l'Océan, il n'en a pas toujours été ainsi. C'est un fait politique nouveau. Chaque collectivité choisit désormais, à son rythme, librement ses institutions, dans les limites de la constitution. La Martinique s'engage ainsi vers la création d'une collectivité autonome tandis que la Guadeloupe se lance dans l'élaboration d'un projet de société partagé. Le congrès des élus départementaux et régionaux, réuni le 18 juin 2009 à Fort-de-France, adopte sept résolutions qui complètent celles qui ont été votées le 18 décembre 2008. Ces résolutions portent sur l'organisation et le fonctionnement (création d'un Conseil territorial et d'un Conseil exécutif), les compétences de la future collectivité (adaptation des lois et règlements, compétences partagées avec l'Etat, compétences supplémentaires propres), la création de conseils consultatifs (conseil des communes et conseil économique, social, culturel, de l'éducation et de l'environnement) et le report des élections régionales prévues en 2010. En Guadeloupe, le congrès des élus départementaux et régionaux, réuni le 24 juin 2009 à Basse-Terre, adopte une seule résolution précisant le calendrier et détaillant les instances et modalités d'élaboration du projet de société. A titre de disposition transitoire, il est également demandé à l'Etat de ne pas engager de projet de modification institutionnelle ou statutaire avant 2011. Simple décalage chronologique ou découplage irrévocable des trajectoires politiques des deux îles?

7 La réponse n'importe. Dans les deux cas, le président Sarkozy retourne à son avantage une situation qui ne lui était guère favorable. Sans relais locaux solides, il parvient ainsi à désamorcer le potentiel contestataire des formations de gauche en reprenant à son compte la demande récurrente des élus en faveur d'une plus grande autonomie interne. En stigmatisant l'immobilisme et le refus du changement, il suscite un alignement original des comportements partisans de la droite ${ }^{4}$. Maître du calendrier de la consultation, c'est aussi lui qui arrêtera les termes de la ou des questions soumises à l'électorat martiniquais. En 2011, c'est encore lui qui décidera des suites éventuelles à donner aux propositions des élus guadeloupéens. En précipitant - au sens chimique du terme - des évolutions en filigrane depuis au moins la fin des années 90, le chef de l'Etat marque sa différence avec ses prédécesseurs, plutôt velléitaires (François Mitterrand), relativement réticents (Jacques Chirac) ou franchement hostiles (Valéry Giscard d'Estaing) à toute évolution institutionnelle ou statutaire des Antilles, alors invariablement interprétée comme l'antichambre d'un desserrement des liens avec la métropole. 


\section{Vers un modèle d'économie endogène et durable}

Dans ce domaine, la rhétorique du président Sarkozy marque également un changement. En combinant « autonomie politique » et «autonomie économique », elle reprend à son compte un modèle de développement préconisé par les formations de gauche depuis les années soixante-dix et invariablement combattu par les partis de droite. Si les premières mettent l'accent sur la nécessité d'un développement " autocentré ", les seconds donnent alors la priorité au jeu de la solidarité nationale. Pour les unes, il s'agit d'appuyer la société sur une économie productive. Pour les autres, il importe avant tout de sanctuariser les transferts sociaux de la métropole. Dans un cas, la coopération régionale est une dimension essentielle du développement. Dans l'autre, le rapport exclusif à la métropole en est le moteur.

9 Au nom du volontarisme et du pragmatisme qui caractérisent sa politique, le chef de l'Etat n'hésite pas à faire bouger les lignes : «Vous le savez, j'ai toujours été et je suis toujours partisan d'un modèle de développement économique endogène qui favorise une plus grande autonomie économique des collectivités d'outre-mer. Pourquoi ? Parce que je pense que l'autonomie économique est la condition de la fierté d'une population au travers de la création de richesses qui sera le fruit de son esprit d'entreprise et de son travail. Et parce que je pense que l'autonomie économique est la condition de toute évolution institutionnelle, évolution pour laquelle, je l'ai toujours dit je n'ai aucun préjugé pour autant qu'elle soit au service d'un projet et que les relations avec la métropole soient clairement définies $»^{5}$. En adoptant ces convictions de la gauche antillaise $^{6}$, le président ne vise pas uniquement l'intérêt général. A l'évidence, son objectif est aussi de «couper l'herbe sous les pieds » de ses opposants locaux dont les plus véhéments tiennent l'Etat responsable de la crise de février 2009. En faisant suite à leurs doléances, il les place devant leurs responsabilités face aux électeurs. Désormais, la balle est dans le camp des parlementaires et élus locaux. Les Etats généraux, mis en œuvre du 15 avril au 31 juillet 2009, permettent de recueillir les "ressentis " et propositions de la «société civile » en vue du futur conseil interministériel de l'Outremer.

10 Pour l'heure, la loi n 2009-594 du 27 mai 2009 pour le développement de l'Outre-mer (LODEOM) répond à des préoccupations plus immédiates en matière de continuité territoriale, soutien au pouvoir d'achat, relance de la politique du logement et soutien à l'économie et aux entreprises. Parmi les mesures prioritaires, le gouvernement a mis l'accent sur la création de zones franches globales d'activités (secteurs émergents et secteurs porteurs d'activités nouvelles), la réorientation progressive de la défiscalisation vers le logement social (jusque-là plutôt orientée vers le secteur immobilier privé), la création d'un fonds exceptionnel d'investissements (destiné aux équipements structurants), la réforme des exonérations de charges pour les recentrer sur les bas salaires. Malgré son adoption retardée ${ }^{7}$, aucune de ces mesures ne rompt avec le tryptique dérogation/subvention/défiscalisation qui constitue le trait d'union de toutes les lois "programme » mises en œuvre Outre-mer depuis près de quarante ans. Par maints aspects, la LODEOM est même en retrait par rapport à la loi Girardin du 21 juillet 2003 qui prévoyait un régime d'incitations fiscales particulièrement avantageux pour les investissements immobiliers Outre-mer. Par ailleurs, la LODEOM n'offre pas de mesures directes visant à réduire les facteurs de vulnérabilité du tissu économique local alors que celui-ci se caractérise par une forte densité de très petites 
entreprises (TPE) dont la mortalité est particulièrement élevée. Le diagnostic est pourtant bien connu. Pour réduire cette "surmortalité », il convient de renforcer les dispositifs d'accompagnement et de formation des chefs d'entreprises et d'étendre Outre-mer les programmes de soutien des TPE existants en métropole. Enfin, aucune mesure directe de la LODEOM ne concerne l'autosuffisance énergétique ou le développement du tourisme haut de gamme. Pourtant, dans ces deux secteurs, les départements antillais disposent d'atouts importants. Dans un rapport récent (Larcher, Doligé, 2009), la mission d'information du Sénat sur la situation des départements d'Outre-mer préconise d'aller plus loin dans l'exploitation et la valorisation des ressources naturelles tout en faisant de l'environnement un élément clé de leur développement endogène.

\section{La consécration d'affirmations identitaires irréductibles}

11 Dans ce domaine hautement symbolique où il était particulièrement attendu, le chef de l'Etat a voulu marquer durablement les esprits. A la veille de son déplacement aux Antilles, rien n'est donc laissé au hasard. Les derniers arbitrages sont rendus. Le programme est arrêté. Les discours sont bouclés. Tout s'enchaîne très vite. Acte 1: nomination au secrétariat d'Etat à l'Outre-mer de Marie-Luce Penchard, conseillère technique à la présidence de la République et fille de Lucette Michaux-Chevry, sénateur de la Guadeloupe. Pour la première fois dans l'histoire de la République, une originaire d'Outre-mer est choisie pour diriger le ministère de la « France des trois océans ». Acte 2 : cérémonie au monument aux morts de Fort-de-France où le président décore plusieurs anciens combattants «oubliés de l'histoire» avant de prononcer une allocution émouvante sur les résistants locaux au régime de Vichy : «Je veux dire aux Martiniquais et aux Guadeloupéens que l'histoire des dissidents est un exemple pour tous les Français et c'est pourquoi j'ai voulu que leur soit rendu l'hommage de toute la nation (...) Je veux dire à ces femmes et à ces hommes que la France n'oublie pas ce qu'elle leur doit $»^{8}$. Là encore, c'est une première dans l'histoire de la République. Une page se tourne. Une injustice est réparée. Acte 3 : baptême de l'aéroport « Aimé Césaire Martinique » où le chef de l'Etat prononce un discours d'hommage particulièrement appuyé et inspiré, citant abondamment le poète mais pas moins l'homme politique, pour affirmer la diversité des Outre-mers et son souci de prendre en compte cette singularité irréductible. Ici aussi, le président va plus loin que ses prédécesseurs en disant ce que la France doit à Aimé Césaire ${ }^{9}$ mais aussi sa volonté d'être à la hauteur de son héritage en renforçant l'autonomie de la Martinique au sein de la République. Acte 4 : bilan d'étape des états généraux de la Guadeloupe où le président a martelé, dans un discours ferme sur les principes mais ouvert sur les modalités, son désir d'être au rendez-vous de la modernisation de la relation des Outre-mers à la République en mettant l'accent sur la nécessité d'une vision partagée de l'avenir de l'archipel. Ce changement passe par l'adaptation des services déconcentrés de l'Etat. Il place aussi les élus locaux devant leurs responsabilités. Il appelle la participation active des Guadeloupéens.

Cette mise en scène n'est évidemment pas sans arrière-pensées politiques. Dans cette course contre la montre, le chef de l'Etat avait plusieurs défis à relever. En premier lieu, il devait faire oublier le rendez-vous raté avec Aimé Césaire alors qu'il était candidat 
aux élections présidentielles. En deuxième lieu, il lui fallait gommer l'image d'un ministre de l'intérieur prompt à la répression dans les banlieues. En troisième lieu, il lui était indispensable de montrer son intérêt et son implication pour sortir de la crise antillaise. Enfin, il lui fallait réinstaller les élus locaux - notamment en Guadeloupe dans leur légitimité d'interlocuteurs privilégiés du Gouvernement et de médiateurs naturels de la population. Sur tous ces plans, ce premier rendez-vous antillais du président semble avoir tenu ses promesses. Celles-ci ne seront toutefois consolidées que si et seulement si le premier conseil interministériel de l'Outre-mer - prévu fin octobre prochain - devait déboucher sur des décisions tangibles.

\section{BIBLIOGRAPHIE}

Constant, F., 2001. « Les catégories politiques du gouvernement Outre-mer » dans Jean-Yves Faberon et alii. dir. La loi d'orientation pour l'Outre-mer de décembre 2000, Paris, Presses Universitaires d'Aix-Marseille, p.15-28.

Daniel, J., 2009. « La crise sociale aux Antilles ». EchoGéo, Sur le vif 2009, http:// echogeo.revues.org/index11117.html

Doligé, E. Larcher, S., 2009. Les DOM, défi pour la République, chance pour la France, 100 propositions pour fonder l'avenir, Paris, rapport d'information n519, 356 pages.

\section{NOTES}

1. Intervention liminaire de M. Le président de la République - Entretien avec les présidents des collectivités locales et les parlementaires des départements d'Outre-mer, Service de Presse de la Présidence de la République, Paris, 2009, 7 pages.

2. A l'occasion notamment de la controverse suscitée par l'article 4 de la loi du 23 février 2005 et du refus consécutif d'Aimé Césaire de recevoir Nicolas Sarkozy, alors ministre d'Etat, ministre de l'intérieur et de l'aménagement du territoire, lors d'un déplacement en Martinique initialement prévu en décembre 2005 et finalement repoussé en mars 2006 après l'abrogation de la disposition en cause. Lire la Lettre aux Antillais du ministre-candidat aux élections présidentielles, publiée dans l'édition du quotidien France-Antilles, du 6 mars 2006, p.3.

3. Ibidem. page 2 .

4. Voir la déclaration de Miguel Laventure, président des Forces Martiniquaises de Progrès, qui se déclare favorable à un rapprochement avec Serge Letchimy, président du Parti Progressiste Martiniquais, sur la question de l'évolution institutionnelle. Cf. France-Antilles Martinique, Fort-deFrance, 28 juin 2009, p.1.

5. Op. Cit.p. 2.

6. Dont certaines remontent jusqu'à la Convention du Morne Rouge signée en 1971 par un ensemble de formations territoriales progressistes des Antilles et de la Guyane.

7. Le projet de loi a été adopté, en Conseil des ministres, le 28 juillet 2008 puis complété, à plusieurs reprises, avant et pendant la discussion parlementaire, pour tenir compte de la gravité de la crise sociale de février-avril 2009. 
8. Allocution du Président de la République, Cérémonie au monument aux morts, Fort-de-France, jeudi 25 juin 2009, p. 5.

9. Voir Constant, F., 2009. « Le pouvoir, la solitude et ...la mort », Esprit, Août, 2009.

\section{RÉSUMÉS}

A l'occasion de son premier déplacement en Martinique et en Guadeloupe, le président Sarkozy a fixé le cap d'une politique aussi volontariste que pragmatique, destinée à faire bouger les lignes du débat public insulaire. Cette nouvelle politique qui précipite des évolutions en filigrane depuis la fin des années 90, repose sur un triptyque : a) affirmer la diversité irréductible des outre-mers et la nécessité de politiques publiques adaptées à chacune de ses composantes b) adapter la gouvernance locale au défi d'un développement endogène et durable c) valoriser les atouts des Outre-mers dans leur environnement régional.

During his first visit to Martinique and Guadeloupe, President SARKOZY set the course for a new pragmatic policy, intended to move the lines of the insular public debate. This policy built on ongoing local evolutions since the end of the 1990s, is aimed at: a) crafting responsive public policies in order to meet the peculiarities of every single Overseas territories; b) adapting local governance to the challenge of a sustainable development; c) developing the assets of the Overseas territories in their regional environment.

\section{INDEX}

Mots-clés : Antilles françaises, gouvernance, identité, politique, Sarkozy

Keywords : French West Indies, identity, local governance, politics, Sarkozy

\section{AUTEUR}

\section{FRED CONSTANT}

Fred Constant est professeur des universités (agrégé de science politique) et chercheur au Centre de Recherches sur les Pouvoirs Locaux dans la Caraïbe (CRPLC, UMR, CNRS 8053). Il a publié récemment : - 2009 : « Construire l'avenir des Outre-mers », (avec Maximin, D. et Vergès, F.) Le Monde, 18 juin, p. 17. - 2009 : « Aimé Césaire. Le pouvoir, la solitude et..la mort », Esprit, août, p. 28-43. - 2009 : « Talking race in color-blind France : equality denied, Blackness reclaimed» dans Keaton, T., Hine, D., Small, S. dir. Black Europe and the African Diaspora, Chicago, University of Illinois Press, p. 268-291. - 2009 : « Pour une gouvernance mondiale des migrations » dans Jaffrelot, C. et Lequesne, C. dir. Les Migrations, Paris, Presses de Sciences-Po-L'Express, Coll. L'enjeu mondial, p. 301-328. fredconstant@hotmail.com 